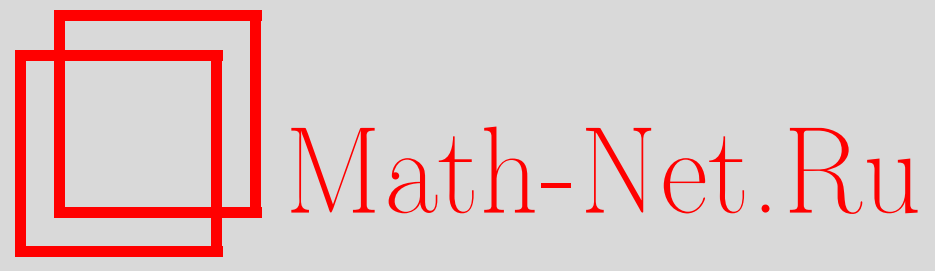

А. Е. Деревянка, Применение различных математических моделей для оценки вероятности столкновения астероидов 99942 Apophis и 2011 AG5 с Землёй, Вестн. Сам. гос. техн. ун-та. Сер. Физ.-мат. науки, 2013, выпуск 4(), 115-121

DOI: https://doi.org/10.14498/vsgtu1256

Использование Общероссийского математического портала MathNet.Ru подразумевает, что вы прочитали и согласны с пользовательским соглашением

http://www.mathnet.ru/rus/agreement

Параметры загрузки:

IP : 54.210 .77 .194

26 апреля 2023 г., $14: 28: 11$

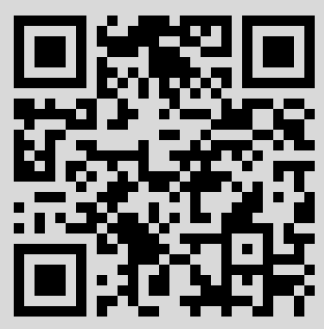


УДК 519.245, 521.1, 521.3

\section{ПРИМЕНЕНИЕ РАЗЛИЧНЫХ МАТЕМАТИЧЕСКИХ МОДЕЛЕЙ ДЛЯ ОЦЕНКИ ВЕРОЯТНОСТИ СТОЛКНОВЕНИЯ АСТЕРОИДОВ 99942 APOPHIS И 2011 AG5 С ЗЕМЛЁЙ}

\section{А. Е. Деревянка}

Самарский государственный технический университет,

Россия, 443100, Самара, ул. Молодогвардейская, 244.

E-mail: andr_derev@mail.ru

Реализовань методъ оценки вероятности столкновения астероидов 99942 Apophis и 2011 AG5 с Землёй. Вероятность столкновения оцениваласъ двумя методами: методом Монте-Карло и как отношение промежутка значений элементов орбит, приводящих $к$ столкновению, $\kappa$ доверителъным интервалам элементов орбит. Установлены элементы орбит, $к$ изменениям в которых орбиты соответствующих астероидов наиболее чувствителъны. Получены предполагаемые даты и оценки вероятности столкновения астероидов с Землёй.

Ключевые слова: 99942 Apophis, 2011 AG5, вероятность столкновения, элементьь орбит, метод Монте-Карло.

На сегодняшний день открыто более 10000 астероидов, которые принадлежат группам Аполлона, Амура и Атона. Свыше 1300 из них проходят через сферу действия Земли на интервале времени с 1800 по 2200 гг. Вследствие тесного сближения с Землёй возможно изменение траектории движения астероида, что может привести к столкновению в будущем. Так как некоторые из астероидов, сближающихся с Землёй в указанном интервале времени, имеют существенные размеры и массу, их можно считать потенциально опасными в силу того, что возмущения, полученные астероидом при тесном сближении с нашей планетой, могут значительно отразиться на его дальнейшей траектории. Таким образом, актуальной задачей является создание математических моделей, позволяющих оценить величину вероятности столкновения астероидов с Землёй. Вследствие того, что элементы орбит вычисляются с определенными погрешностями, важно оценить влияние этих погрешностей на величину вероятности столкновения этого астероида с Землёй. Также необходимо оценить и саму вероятность столкновения в будущем.

Астероид 99942 Apophis был открыт 19 июня 2004 года. По данным Лаборатории реактивного движения NASA, наиболее тесное сближение данного объекта с Землёй произойдет 13 апреля 2029 г., когда Апофис приблизится к Земле на 0,000256 а.е. (38,3 тыс. км). Вследствие тесного сближения с Землёй орбита астероида существенно изменится, поэтому не исключена вероятность его столкновения с Землёй в будущем. Диаметр астероида Апофис составляет 320 м, масса $-2,7 \cdot 10^{10} \mathrm{kг}$, поэтому он является потенциально опасным для Земли объектом.

Астероид 2011 AG5 был обнаружен 8 января 2011 года. По данным Лаборатории реактивного движения NASA, астероид сблизится с Землёй 4 февраля 2040 г. и пройдет на геоцентрическом расстоянии в 0,0072 а.е. Диаметр

Андрей Евгенвевич Деревянка, аспирант, каф. прикладной математики и информатики. 
астероида 2011 AG5 - 140 м, приблизительная масса 4,1 $10^{9}$ кг. Первоначально у данного астероида элементы орбит были вычислены с большими погрешностями, что позволило отнести его к потенциально опасным объектам.

Чтобы оценить вероятность столкновения астероидов с Землёй, использовались две математические модели: в основе одной лежит метод статистических испытаний (метод Монте-Карло [1]), вторая же основана на отношении мер областей (геометрическое определение вероятности наступления события).

Положение астероида в пространстве однозначно определяют элементы орбит. Это шесть элементов, два из которых (большая полуось $a$ и эксцентриситет $e$ ) задают форму орбиты, три (наклонение $i$, долгота восходящего узла $\Omega$ и аргумент перигелия $\omega)$ - ориентацию по отношению к базовой системе координат, и один (средняя аномалия $M$ ) определяет положение тела на орбите.

Источником значений элементов орбит на различные даты наблюдений являлся сайт-каталог орбитальной эволюции малых тел Солнечной системы smallbodies.ru. Начальные данные для астероидов 99942 Apophis и 2011 AG5 приведены в табл. 1 и табл. 2 соответственно.

В качестве математической модели движения астероидов были использованы дифференциальные уравнения движения с учётом гравитационных и релятивистских эффектов от Солнца, имеющие в гелиоцентрической системе координат следующий вид [2]:

Таблица 1

Элементы орбит астероида 99942 Apophis на различные моменты наблюдений

\begin{tabular}{|c|c|c|c|}
\hline Әлемент Дата & 06.03 .2006 & 04.01 .2010 & 27.08 .2011 \\
\hline$a$, a.e. & 0,92239593 & 0,92241929 & 0,92230028 \\
\hline$e$ & 0,19104000 & 0,19121109 & 0,19107611 \\
\hline$i$, град. & 3,331224 & 3,331512 & 3,331952 \\
\hline$\Omega$, град. & 204,462302 & 204,439306 & 204,430424 \\
\hline$\omega$, град. & 126,355659 & 126,424463 & 126,424469 \\
\hline М, град. & 222,272876 & 339,948637 & 287,582163 \\
\hline
\end{tabular}

Таблица 2

Элементы орбит астероида 2011 AG5 на различные моменты наблюдений

\begin{tabular}{|c|c|c|c|}
\hline Әлемент Дата & 19.05 .2011 & 27.08 .2011 & 05.12 .2011 \\
\hline$a$, a.e. & 1,43063113 & 1,43048221 & 1,43056303 \\
\hline$e$ & 0,39056001 & 0,39049892 & 0,39041690 \\
\hline$i$, град. & 3,680462 & 3,680517 & 3,680351 \\
\hline$\Omega$, град. & 135,713596 & 135,712206 & 135,710898 \\
\hline$\omega$, град. & 53,480619 & 53,473598 & 53,479691 \\
\hline М, град. & 32,077136 & 89,695558 & 147,300586 \\
\hline
\end{tabular}




$$
\begin{aligned}
\frac{d^{2} X}{d t^{2}} & =-k^{2}(1+m) \frac{X}{r^{3}}+\sum_{i} k^{2} m_{i}\left(\frac{X_{i}-X}{\Delta_{i}^{3}}-\frac{X_{i}}{r_{i}^{3}}\right)+ \\
& +\frac{k^{2}}{c^{2}}\left[(4-2 \alpha) \frac{k^{2}}{r^{2}} X-(1+\alpha) \frac{\dot{r}^{2}}{r^{3}} X+3 \alpha \frac{(X \dot{X})^{2}}{r^{5}} X+(4-2 \alpha) \frac{(X \dot{X})}{r^{3}} \dot{X}\right],
\end{aligned}
$$

где $X$ - матрица-столбец с элементами $x, y, z ; X_{i}$ - матрица-столбец с элементами $x_{i}, y_{i}, z_{i} ; m, x, y, z$ - масса и гелиоцентрические координаты астероида; $m_{i}, x_{i}, y_{i}, z_{i}$ - массы и гелиоцентрические координаты больших планет; $r, \Delta_{i}, r_{i}$ - расстояния, вычисляемые по формулам: $r^{2}=x^{2}+y^{2}+z^{2}$, $\Delta=\left(x_{i}-x\right)^{2}+\left(y_{i}-y\right)^{2}+\left(z_{i}-z\right)^{2}, r_{i}^{2}=x_{i}^{2}+y_{i}^{2}+z_{i}^{2} ; \dot{X}$ - матрица-столбец с элементами $\dot{x}, \dot{y}, \dot{z} ; k$ - постоянная Гаусса; $c$ - скорость света; $\alpha$ - параметр, характеризующий выбор системы координат. Случай при $\alpha=1$ соответствует стандартным координатам, при $\alpha=0$ - гармоническим координатам.

Для рассматриваемой задачи (оценка величины вероятности столкновения астероида с Землёй) сущность метода Монте-Карло состоит в следующем: генерируется определённое количество виртуальных астероидов, элементы орбит которых распределены в пределах области возможных начальных значений, определяемой значениями математических ожиданий элементов орбит и ковариационной матрицей. Затем с заданной точностью численного интегрирования прослеживается движение каждого виртуального астероида на определенном временном отрезке, чтобы установить, произойдет ли столкновение этого объекта с Землёй. Если в определенный момент времени расстояние между центрами планеты и астероида становится меньше радиуса Земли $(6378 \mathrm{kM})$, то считается, что произошло столкновение и интегрирование уравнений движения для этого виртуального астероида прекращается. При большом количестве испытаний отношение числа виртуальных астероидов, для которых выполнилось условие столкновения, к общему числу испытаний может служить оценкой вероятности столкновения [3]. Метод Монте-Карло является сходящимся по вероятности [1].

Для расчёта по методу Монте-Карло для каждого из астероидов (99942 Apophis и 2011 AG5) было сгенерировано 30000 виртуальных астероидов с учетом корреляционных связей между элементами орбит. Ковариационная матрица, отражающая зависимости между элементами орбит каждого астероида, была взята с сайта Лаборатории реактивного движения NASA (neo.jpl . nasa.gov). Для генерации начальных данных виртуальных астероидов использовался Вихрь Мерсенна. Так как в основе данного генератора лежат свойства простых чисел Мерсенна, Вихрь Мерсенна обладает внушительным периодом, равным числу Мерсенна $2^{19937}-1$. Это позволяет минимизировать корреляцию между последовательными значениями в выходной последовательности псевдослучайных величин [4]. В качестве математических ожиданий принимались данные наблюдений, приведённые в табл. 1 и табл. 2, для которых среднеквадратические отклонения приведены в табл. 3 и табл. 4. Уравнения движения астероидов интегрировались методом Эверхарта 27 порядка на интервале времени с 2006 по 2040 гг. [5, 6].

Было установлено, что орбиты обоих астероидов чувствительны к возмущению начальных данных, так как при варьировании элементов орбит и последующем интегрировании была обнаружена возможность столкновения: 
Таблица 3

Величины допустимых отклонений элементов орбиты для 99942 Apophis

\begin{tabular}{|c|c|c|c|c|c|c|}
\hline Элемент & $a$, a.e. & $e$ & $i$, град. & $\Omega$, град. & $\omega$, град. & М, град. \\
\hline$\sigma$ & $9,6 \cdot 10^{-9}$ & $1,0 \cdot 10^{-7}$ & $3,0 \cdot 10^{-6}$ & $1,5 \cdot 10^{-4}$ & $1,5 \cdot 10^{-4}$ & $3,0 \cdot 10^{-6}$ \\
\hline
\end{tabular}

Таблица 4

Величины допустимых отклонений элементов орбиты для 2011 AG5

\begin{tabular}{|c|c|c|c|c|c|c|}
\hline Элемент & $a$, a.e. & $e$ & $i$, град. & $\Omega$, град. & $\omega$, град. & M, град. \\
\hline$\sigma$ & $6,14 \cdot 10^{-6}$ & $3,35 \cdot 10^{-6}$ & $3,1 \cdot 10^{-5}$ & $7,8 \cdot 10^{-5}$ & $1,1 \cdot 10^{-4}$ & $1,3 \cdot 10^{-6}$ \\
\hline
\end{tabular}

для астероида 99942 Apophis - 13 апреля 2037 г., а для 2011 AG5 - 5 февраля 2040 г., так как некоторые из сгенерированных астероидов сталкиваются с Землёй.

Реализация метода Монте-Карло относительно проста, но трудоёмка: чем меньше вероятность события, тем больше экспериментов необходимо провести для получения достоверной оценки вероятности. Вероятность столкновения астероида с Землёй может быть оценена как отношение промежутка значений элементов орбит, приводящих к столкновению, к доверительным интервалам элементов орбит. Начальные данные элементов орбит астероида на различные моменты оскуляции варьировались в пределах их доверительных интервалов, а затем производилось интегрирование уравнений движения астероида. При этом для каждого элемента оценивались величина и дата сближения с Землёй. Столкновение засчитывалось, если расстояние между центрами Земли и астероида меньше радиуса Земли. Для получения области возможных значений элементов орбит предполагалось, что параметры орбиты являются нормально распределенными случайными величинами с математическими ожиданиями, равными данным наблюдений. Величины допустимых отклонений значений элементов орбит принимались равными указанным на сайте Лаборатории реактивного движения NASA (neo.jpl.nasa.gov) и приведены в табл. 3 и 4 для астероидов 99942 Apophis и 2011 AG5 соответственно.

В результате удалось установить, что определяющим элементом, влияющим на величину вероятности столкновения, для астероида 99942 Apophis является большая полуось (параметр а), а для астероида 2011 AG5 такими элементами являются большая полуось и эксцентриситет (параметр e), так как их изменения вносят наибольший вклад в оценку величины вероятности столкновения.

Вероятность столкновения астероида 99942 Apophis с Землёй (событие $A$ ) может быть оценена как отношение опасного промежутка значений $a$ к доверительному интервалу:

$$
P(A)=\frac{\left|a_{1}-a_{2}\right|}{6 \sigma_{a}},
$$

где $\sigma_{a}$ - среднеквадратическое отклонение для большой полуоси.

Вероятность столкновения астероида 2011 AG5 с Землёй (событие $A$ ) можно оценить как отношение суммы площадей областей, в которых находятся параметры (а) и е, приводящие к столкновению, к площади доверительной 
области, ограничивающей значения системы величины $(a, e)$, по формуле

$$
P(A)=\sum_{i} S_{i} / D,
$$

где $S_{i}$ - площадь $i$-той опасной области, $D$ - площадь доверительной области (доверительный эллипс) для системы случайных величин $(a, e)$.

Оценки вероятности столкновения, полученные в данной работе различными методами, для астероида 99942 Apophis приведены в табл. 5, а для 2011 AG5 - в табл. 6.

Таблица 5

Оценки величины вероятности столкновения 13 апреля 2037 г., полученные различными методами для астероида 99942 Apophis

\begin{tabular}{|c|c|c|}
\hline Дата наблюдений & Монте-Карло & Отношение промежутков \\
\hline 06.03 .2006 & $2,33 \cdot 10^{-4}$ & $3,41 \cdot 10^{-4}$ \\
\hline 04.01 .2010 & $2,67 \cdot 10^{-4}$ & $3,37 \cdot 10^{-4}$ \\
\hline 27.08 .2011 & $1,67 \cdot 10^{-4}$ & $3,99 \cdot 10^{-4}$ \\
\hline
\end{tabular}

Таблица 6

Оценки величины вероятности столкновения 5 февраля 2040 г., полученные различными методами для астероида 2011 AG5

\begin{tabular}{|c|c|c|}
\hline Дата наблюдений & Монте-Карло & Отношение областей \\
\hline 19.05 .2011 & $2,00 \cdot 10^{-4}$ & $1,19 \cdot 10^{-4}$ \\
\hline 27.08 .2011 & $1,33 \cdot 10^{-4}$ & $2,80 \cdot 10^{-4}$ \\
\hline 05.12 .2011 & $1,00 \cdot 10^{-4}$ & $0,91 \cdot 10^{-4}$ \\
\hline
\end{tabular}

Для каждого астероида оценки, полученные различными методами, согласуются между собой и расположены достаточно близко друг к другу. Как можно заметить, оценки вероятности столкновения на различные даты наблюдений различаются, но при этом не выходят за границы определенной области, что позволяет судить о непротиворечивости полученных результатов для каждого из астероидов.

Оценка вероятности столкновения астероида 99942 Apophis ранее была рассмотрена в статье [7], однако в ней не была учтена корреляция элементов орбиты астероида при использовании метода Монте-Карло. В данной работе корреляция учитывается посредством использовании ковариационной матрицы при генерации элементов орбит для виртуальны астероидов, что и позволяет получить оценки, отличные от полученных в работе [7].

Оценка величины вероятности и дата столкновения (13 апреля 2037 г. для 99942 Apophis и 5 февраля 2040 г. для 2011 AG5) для начальных данных на приведенные даты наблюдений не противоречат результатам других исследований. Так, по данным Лаборатории реактивного движения NASA, оценка вероятности столкновения астероида 99942 Apophis с Землёй равна $4,5 \cdot 10^{-4}$ [8], а астероида 2011 AG5 - 2,0 $\cdot 10^{-3}$. Кроме того, в статье [9] предложена оценка вероятности столкновения астероида 99942 Apophis, равная $2,5 \cdot 10^{-5}$, а в статье [10] - 1,56 $\cdot 10^{-5}$ для астероида 2011 AG5. Для вычисления указанных выше оценок (за исключением оценки, предложенной в работе [9], где 
применялся метод, основанный на интервальной арифметике), использовался метод Монте-Карло с учётом корреляционных связей между элементами орбит. Значительное отличие оценки, предложенной в статье [9], от оценок, полученных в данной работе, может быть обусловлено как использованием различных начальных данных, так и различием численных методов, использованных для интегрирования уравнений движения (в работе [9] применялись методы, усовершенствованные для использования интервальной арифметики). Стоит отметить, что оценки для астероида 2011 AG5 приведены на март 2012 года и являются весьма высокими. Однако после уточнения начальных данных и пересчёта орбиты этот астероид был исключен из списка потенциально опасных. На данный момент он не представляет опасности, что отражает значимость точности начальных данных для моделирования движения астероидов.

Работа выполнена при финансовой поддержке Министерства образования и науки РФ (проект РНП 2.534.2011: «Разработка математического и программного обеспечения для исследования эволюции орбит главных метеорных потоков»).

\section{БИБЛИОГРАФИЧЕСКИЙ СПИСОК}

1. C. М. Ермаков, Метод Монте-Карло и смежные вопросы. М.: Наука, 1975. 472 с. [S. M. Ermakov, The Monte Carlo method and related problems. Moscow: Nauka, 1975. 472 pp.]

2. В. А. Брумберг, Релятивистская небесная механика. М.: Наука, 1972. 382 с. [V. A. Brumberg, Relativistic celestial mechanics. Moscow: Nauka, 1972. 382 pp.]

3. Н. Б. Железнов, "Влияние корреляционных связей между оцениваемыми по наблюдениям орбитальными параметрами астероида на результаты определения вероятности его столкновения с планетой методом Монте-Карло" // Астрономический вестник, 2010. Т. 44, № 2. С. 150-157; англ. пер.: N. B. Zheleznov, "The influence of the correlations between an asteroid's orbital parameters on the estimation of the probability of planetary collision by the Monte Carlo method" // Solar System Research, 2010. Vol. 44, no. 2. Pp. 136143.

4. M. Matsumoto, T. Nishimura, "Mersenne twister: A 623-dimensionally equidistributed uniform pseudorandom number generator" // ACM Transactions on Modeling and Computer Simulation (TOMACS), 1998. Vol. 8, no. 1. Pp. 3-30.

5. E. Everhart, "Implicit single-sequence methods for integrating orbits" // Celestial mechanics, 1974. Vol. 10, no. 1. Pp. 35-55.

6. А. Ф. Заусаев, А. А. Заусаев, "Применение модифицированного метода Эверхарта для решения задач небесной механики" // Матем. моделирование, 2008. Т. 20, № 11. С. 109114. [A. F. Zausaev, A. A. Zausaev, "Employment of the modification Everhart's method for solution of problems of celestial mechanics" // Matem. Mod., 2008. Vol.20, no.11. Pp. 109114].

7. А. Ф. Заусаев, А. Е. Деревянка, "Сравнительный анализ математических моделей для оценки вероятности столкновения астероида Апофис с Землёй” // Вестн. Сам. гос. техн. ун-та. Сер. Физ.-мат. науки, 2012. №2(27). С. 192-196. [A. F. Zausaev, A. E. Derevyanka, "Comparative analysis of mathematical models for estimating the impact probability of asteroid Apophis" // Vestn. Samar. Gos. Tekhn. Univ. Ser. Fiz.-Mat. Nauki, 2012. no. 2(27). Pp. 192-196].

8. J. D. Giorginia, L. A. M. Benner, S. J. Ostro, M. C. Nolan, M. W. Busch, "Predicting the Earth encounters of (99942) Apophis" // Icarus, 2008. Vol. 193, no. 1. Pp. 1-19.

9. E. А. Смирнов, "Использование интервальной арифметики при прогнозировании орбит малых тел" / В сб.: Астрономия и всемирное наследие через время и континенты: Труды международной конференции. Секиия «Околоземная астрономия». Казань: Казан. 
гос. ун-т., 2009. С. 101-102. [E. A. Smirnov, "The use of interval arithmetic in predicting the orbits of small bodies" / In: Astronomy and World Heritage across time and continents: Proc. Internat. Conf. Section of Near-Earth Astronomy. Kazan: Kazan Federal University, 2009. Pp. 101-102].

10. D. R. Adamo, Earth Risk Corridor Computations for 2011 AG5 on 5 February 2040, 3 March 2012 (http://astrogatorsguild.com/wp-content/uploads/2012/03/ RiskCorr2011-AG5r2.pdf).

Поступила в редакцию 21/V/2013;

в окончательном варианте - 25/VIII/2013.

MSC: 85-08; 70M20, 65C05

\section{APPLICATION OF THE MATHEMATICAL MODELS USED FOR ESTIMATING THE IMPACT PROBABILITY OF ASTEROIDS 99942 APOPHIS AND 2011 AG5}

\section{A. E. Derevyanka}

Samara State Technical University,

244, Molodogvardeyskaya st., Samara, 443100, Russia.

E-mail: andr_derev@mail.ru

Methods for estimating the impact probability of asteroids 99942 Apophis and 2011 AG5 were implemented. The probability of a collision was assessed using two methods: The Monte Carlo method (statistical experiments), and as a ratio between the interval of values of orbital elements, leading to a collision, and the confidence intervals of orbital elements. It was established that the main contribution to the estimation of the impact probability makes a variation in the semimajor axis. The expected date and the estimate of the probability of collisions of asteroids 99942 Apophis and 2011 AG5 with the Earth were computed.

Keywords: 99942 Apophis, 2011 AG5, impact probability, orbital elements, Monte Carlo method.

Original article submitted $21 / \mathrm{V} / 2013$ revision submitted 25/VIII/2013.

Andrey E. Derevyanka, Postgraduate Student, Dept. of Applied Mathematics \& Computer Science. 